\title{
On the Complex Inversion Formula and Admissibility for a Class of Volterra Systems
}

\author{
Ahmed Fadili and Hamid Bounit \\ Department of Mathematics, Faculty of Sciences, Ibn Zohr University, BP 8106, 8000 Agadir, Morocco \\ Correspondence should be addressed to H. Bounit; bounith@yahoo.fr
}

Received 13 January 2014; Revised 13 April 2014; Accepted 26 April 2014; Published 1 June 2014

Academic Editor: J. M. A. M. van Neerven

Copyright ( 92014 A. Fadili and H. Bounit. This is an open access article distributed under the Creative Commons Attribution License, which permits unrestricted use, distribution, and reproduction in any medium, provided the original work is properly cited.

This paper studies Volterra integral evolution equations of convolution type from the point of view of complex inversion formula and the admissibility in the Salamon-Weiss sens. We first present results on the validity of the inverse formula of the Laplace transform for the resolvent families associated with scalar Volterra integral equations of convolution type in Banach spaces, which extends and improves the results in Hille and Philllips (1957) and Cioranescu and Lizama (2003, Lemma 5), respectively, including the stronger version for a class of scalar Volterra integrodifferential equations of convolution type on unconditional martingale differences UMD spaces, provided that the leading operator generates a $C_{0}$-semigroup. Next, a necessary and sufficient condition for $L^{p}$-admissibility $(p \in[1, \infty[)$ of the system's control operator is given in terms of the UMD-property of its underlying control space for a wider class of Volterra integrodifferential equations when the leading operator is not necessarily a generator, which provides a generalization of a result known to hold for the standard Cauchy problem (Bounit et al., 2010, Proposition 3.2).

\section{Introduction}

The purpose of this paper is to analyze conditions for the inversion formula and the $L^{p}$-admissibility for control operators for the solution of the following integrodifferential equation:

$$
\begin{gathered}
\dot{x}(t)=A x(t)+\int_{0}^{t} k(t-s) A x(s) d s+B u(t), \quad t \geq 0, \\
x(0)=x_{0} \in X,
\end{gathered}
$$

which has a "big" intersection with the class of scalar Volterra integral equations. Here we assume that $A$ is a closed linear densely defined operator in a Banach space $X$, and the kernel $k$ belonging to $L_{\text {loc }}^{1}\left(\mathbb{R}^{+}\right)$is real-valued and of at most exponential growth. $B$ is a (possibly unbounded) linear operator on another Banach space $U$ and the control function $u \in L_{\mathrm{loc}}^{p}\left(\mathbb{R}^{+} ; U\right)$.
It is further assumed that the uncontrolled system, that is,

$$
\begin{gathered}
\dot{x}(t)=A x(t)+\int_{0}^{t} k(t-s) A x(s) d s, \quad t \geq 0, \\
x(0)=x_{0},
\end{gathered}
$$

is well-posed, which is equivalent to the existence of a unique family of bounded linear operators $(S(t))_{t \geq 0}$ on $X$ called the resolvent or solution family for (2), where we write $(S(t))_{t \geq 0} \subset$ $\mathscr{L}(X)$ ) (see Section 2 ) and that $(S(t))_{t \geq 0}$ is exponentially bounded.

Many authors have studied this class of Volterra integral equations by the classical approach and for different reasons, using the Laplace transform (see, e.g., Da Prato and Iannelli $[1,2]$, Grimmer and Pritchard [3], Lunardi [4], and Prüss [5, 6]). Maximal $-L^{p}$ regularity results are due to Clément and $\mathrm{Da}$ Prato [7] and Prüss [6]. In addition to the classical approach, there is a semigroup approach which was used in, for example, Miller [8], Chen and Grimmer [9, 10], Desch and Grimmer [11], Desch and Schappacher [12], Di Blasio et al. [13], 
Nagel and Sinestrari [14], and Engel and Nagel [15]. It was the main objection against the semigroup approach for many years that it is not possible to obtain regularity of the solutions. This is not true, as it was proved recently in [16].

From the point of view of complex inversion formula, early Hille and Philllips have proved in [17, p. 349] the validity of the complex inversion formula of the Laplace transform for $C_{0}$-semigroups (i.e., (2) with $k(t)=0$ ) on the domain $D(A)$. In 1995, Yao has proved [18], in Hilbert spaces, the validity of the complex inversion formula of the Laplace transform for $C_{0}$-semigroups on $X$. In 1999, Driouich and ElMennaoui have proved [19] (see also [20, Proposition 3.12.2]) that this inversion result remains true on UMD spaces. These results have been extended to strongly continuous cosine families by Cioranescu and Keyantuo in [21] and to strongly continuous resolvent families by Cioranescu and Lizama in [22]. Recently, Haase has improved some results in [23] based on Fourier analysis and left as an open problem the corresponding result for convoluted semigroups, which has been solved affirmatively very recently in [24].

Several authors have been investigating the Cauchy problem from the point of view of admissibility of control operators (i.e., (2) with $k(t)=0$ ) in the past and the present [25-32] et al. But the first studies on $L^{2}$-admissibility of control operator for Volterra integral scalar systems began with the paper of Jung [33]. The idea of treating $L^{2}$-admissibility for Volterra integral equations has been exploited in the past years by several authors, for example, [34-36]. In [33], the notion of finite-time $L^{2}$-admissibility for Volterra integral scalar system is linked with finite-time admissibility of the well-studied semigroups' (i.e., $k(t)=0$ ) case for completely positive kernel. Likewise, in [34] infinite-time admissibility for a Volterra scalar system is linked with infinite-time admissibility for semigroups (i.e., $k(t)=0$ ) for a large class of kernels and the result subsumes that of [33]. Other results are related to the case where the generator of the underlying semigroup has a Riesz basis of eigenvectors in [36]. In [35], the authors have given necessary and sufficient conditions for finite-time $L^{2}$ admissibility of linear Volterra integrodifferential systems (2) when the underlying semigroup is equivalent to a contraction semigroup, which generalizes an analogous result known to hold for the standard Cauchy problem and it subsumes the result in [33]. Recently, the authors in [37] have introduced the notion of Favard spaces with respect to resolvent families and have established a relationship between $L^{p_{-}}$ admissibilities to these Favard spaces. This extends the results obtained for the semigroups' case in [32]. Furthermore, it was proved in [37] that, for scalar Volterra integral systems with a creep kernel, finite- and infinite-time $L^{1}$-admissibility are equivalent to exponentially stable resolvent family, and if the state space $X$ is reflexive then finite-time and uniformly finite-time $L^{1}$-admissibility are equivalent, extending wellknown results for semigroups.

We proceed as follows. In Section 2, we review some wellknown properties of resolvent families for scalar Volterra integral equations and their properties. Section 3 contains the definition of the UMD space and recalls some results on the complex inversion formula for wide classes of families of bounded linear operators on UMD spaces and prove the analogue of [17, Theorem 11.6.2] which is applied in Sections 4 and 5. Our hypotheses on the kernel differ from those considered by $[22,23]$ and can contain a class of completely positive functions (see [35, Example 4.5]). In Section 4, we are concerned with a class of scalar integrodifferential Volterra equations. First we embed this class in a larger Cauchy system, a technique originating in Engel and Nagel [15, VI.7], in order to prove some results concerning the validity of the complex inversion formula. In Section 5, we go back to the study of the admissibility of control operators for Volterra integrodifferential equations (1) in the same spirit of semigroups and we get a new criterion to judge $L^{p}$ admissibility $(p \in[1, \infty[$ ) of control operators in terms of UMD property of its underlying control space. If we set $k(t)=$ 0 then we recover the result in [38] for the semigroups. Note that this paper involves in particular a nonscalar kernel of the form " $K(t)=k(t) A$ " and so a natural question is whether the situation extends when $K(t)$ is a nonscalar kernel. In a forthcoming work, we will consider a class of nonscalar kernels.

\section{Review on Resolvent Families}

In this subsection, we collect some elementary facts about scalar Volterra integral equations and resolvent families. These topics have been covered in detail in [6]. We refer to these works for reference to the literature and further results.

Let $\left(X,\|\cdot\|_{X}\right)$ be a Banach space; let $A$ be a linear closed densely defined operator in $X ; a \in L_{\text {loc }}^{1}\left(\mathbb{R}^{+}\right)$is a scalar kernel. We consider the linear Volterra integral equation

$$
\begin{gathered}
x(t)=x_{0}+\int_{0}^{t} a(t-s) A x(s) d s, \quad t \geq 0, \\
x(0)=x_{0} \in X .
\end{gathered}
$$

We denote by $[D(A)]$ the domain of $A$ equipped with the graph norm.

We define the convolution product of the scalar function $a$ with a vector-valued function $f$ by

$$
(a * f)(t):=\int_{0}^{t} a(t-s) f(s) d s, \quad t \geq 0 .
$$

Definition 1. A function $x \in C\left(\mathbb{R}^{+}, X\right)$ is called

(i) strong solution of (3) if $x \in C\left(\mathbb{R}^{+},[D(A)]\right)$ and (3) is satisfied,

(ii) mild solution of (3) if $a * x \in C\left(\mathbb{R}^{+},[D(A)]\right)$ and

$$
x=x_{0}+A[a * x](t), \quad t \geq 0 .
$$

Obviously, every strong solution of (3) is a mild solution. Conditions under which mild solutions are strong solutions are studied in [6].

Definition 2. Equation (3) is called well-posed if, for each $v \in$ $D(A)$, there is a unique strong solution $x(t, v)$ on $\mathbb{R}^{+}$of

$$
x(t, v)=v+(a * A x)(t), \quad t \geq 0,
$$


and, for a sequence $\left(x_{n}\right) \subset D(A), x_{n} \rightarrow 0$ implies $x\left(t, x_{n}\right) \rightarrow$ 0 in $X$, uniformly on compact intervals.

Definition 3. Let $a \in L_{\text {loc }}^{1}\left(\mathbb{R}^{+}\right)$. A strongly continuous family $(S(t))_{t \geq 0} \subset \mathscr{L}(X)$ is called resolvent family for (3), if the following three conditions are satisfied:

(S1) $S(0)=I$;

(S2) $S(t)$ commutes with $A$, which means that $S(t)$ $(D(A)) \subset D(A)$, for all $t \geq 0$, and $A S(t) x=S(t) A x$ for all $x \in D(A)$ and $t \geq 0$;

(S3) for each $x \in D(A)$ and all $t \geq 0$ the resolvent equations hold:

$$
S(t) x=x+\int_{0}^{t} a(t-s) S(s) A x d s .
$$

Note that the resolvent for (3) is uniquely determined. The proofs of these results and further information on resolvent can be found in the monograph by Prüss [6]. We also notice that the choice of the kernel a classifies different families of strongly continuous solution operators in $\mathscr{L}(X)$. For instance, when $a(t)=1$, then $S(t)$ corresponds to a $C_{0^{-}}$ semigroup and when $a(t)=t$, then $S(t)$ corresponds to cosine operator function. In particular, when $a(t)=t^{\alpha-1} / \Gamma(\alpha)$ with $0<\alpha \leq 2$, they are the $\alpha$-times resolvent families studied by [39] and correspond to the solution families for fractional evolution equations, that is, evolution equations where the integer derivative with respect to time is replaced by a derivative of fractional order.

The existence of a resolvent family allows one to find the solution for (3). Several properties of resolvent families have been discussed in $[6,40]$.

The following well-known result [6, Proposition 1.1] establishes the relation between well-posedness and existence of a resolvent family.

Theorem 4. Equation (3) is well-posed if and only if (3) admits a resolvent family $(S(t))_{t \geq 0}$. If this is the case one has in addition Range $(a * S)(t) \subset D(A)$, for all $t \geq 0$, and

$$
S(t) x=x+A \int_{0}^{t} a(t-s) S(s) x d s
$$

for each $x \in X, t \geq 0$.

From this, we obtain that if $(S(t))_{t \geq 0}$ is a resolvent family of (3), we have $A(a * S)(\cdot)$ which is strongly continuous and the so-called mild solution $x(t)=S(t) x_{0}$ solves (3).

A resolvent family $(S(t))_{t \geq 0}$ is called exponentially bounded, if there exist $M>0$ and $\omega \in \mathbb{R}$ such that $\|S(t)\| \leq$ $M e^{\omega t}$ for all $t \geq 0$, and the pair $(M, \omega)$ is called type of $(S(t))_{t \geq 0}$. The growth bound of $(S(t))_{t \geq 0}$ is $\omega_{0}(S):=\inf \{\omega \in$ $\left.\mathbb{R},\|S(t)\| \leq M e^{\omega t}, t \geq 0, M>0\right\}$. The resolvent family is called exponentially stable if $\omega_{0}(S)<0$.

Note that, contrary to the case of $C_{0}$-semigroup, the resolvent for (3) needs not to be exponentially bounded; a counterexample can be found in $[6,41]$. However, there are checkable conditions guaranteeing that (3) possesses an exponentially bounded resolvent operator.

We will use the Laplace transform at times. Suppose $g$ : $\mathbb{R}^{+} \rightarrow X$ is measurable and there exist $M>0$ and $\omega \in \mathbb{R}$, such that $\|g(t)\| \leq M e^{\omega t}$ for almost $t \geq 0$. Then, the Laplace transform

$$
\widehat{g}(\lambda)=\int_{0}^{\infty} e^{-\lambda t} g(t) d t
$$

exists for all $\lambda \in \mathbb{C}$ with $\operatorname{Re} \lambda>\omega$.

The following well-known generation result, stated in [6], is quite important in this paper. It establishes the relation between resolvent family and Laplace transform.

Proposition 5. Let $a \in L_{\text {loc }}^{1}\left(\mathbb{R}^{+}\right)$be $\omega$-exponentially bounded. Then, (3) admits a resolvent family $(S(t))_{t \geq 0}$ of type $(M, \omega)$ if and only if the following conditions hold:

(i) $\widehat{a}(\lambda) \neq 0$ and $1 / \widehat{a}(\lambda) \in \rho(A)$ (the resolvent set of $A$ ), for all $\lambda>\omega$;

(ii) $H(\lambda):=(1 / \lambda \widehat{a}(\lambda))((1 / \widehat{a}(\lambda)) I-A)^{-1}$ called the resolvent associated with $(S(t))_{t \geq 0}$ satisfies

$$
\left\|H^{(n)}(\lambda)\right\| \leq M n !(\lambda-\omega)^{-(n+1)} \quad \forall \lambda>\omega, n \in \mathbb{N} .
$$

Under these assumptions the Laplace transform of $S(\cdot)$ is well-defined and it is given by $\widehat{S}(\lambda)=H(\lambda)$ for all $\operatorname{Re}(\lambda)>$ $\omega$. Note that, for $a(t)=1$, Proposition 5 becomes the wellknown Hille-Yosida theorem.

\section{The Complex Inversion Formula and UMD Spaces}

In this section, we review some results on the complex inversion formula of the Laplace transform, in the strong sense, for wide classes of families of bounded linear operators on UMD spaces. There are several equivalent definitions of a UMD space, one of which involves the so-called unconditional Martingale differences, but we will use a different characterization, due to [42-44] involving the vector-valued Hilbert transform (see [45]) for more about UMD spaces. by

Let $p \in] 1, \infty\left[\right.$, and define the operator $\mathscr{H}_{\varepsilon}$ on $L^{p}(\mathbb{R} ; X)$

$$
\mathscr{H}_{\varepsilon} f(t):=\frac{1}{\pi} \int_{|t-s| \geq \varepsilon} \frac{f(s)}{t-s} d s \quad \forall t \in \mathbb{R} .
$$

A Banach space $X$ is called a UMD space (or said to have the UMD-property) if for some (and hence all) $p \in] 1, \infty[$ (see $[44,46]) \mathscr{H} f:=\lim _{\varepsilon \downarrow 0} \mathscr{H}_{\varepsilon} f$ exists in $L^{p}(\mathbb{R} ; X)$ and defines a bounded operator $\mathscr{H}$ on $L^{p}(\mathbb{R} ; X)$. The operator $\mathscr{H}$ is called the Hilbert transform on $L^{p}(\mathbb{R} ; X)$. Every UMD space is reflexive and its dual is also a UMD space. Typical examples of UMD spaces are $L^{p}(\Omega)$-spaces, Sobolev spaces $W_{p}^{s}(\Omega)$, and Besov spaces $B_{p, q}^{s}$ for $\left.p, q \in\right] 1, \infty[$ and their closed subspaces.

The natural question that comes in mind is the following: let $S(t)_{t \geq 0}$ be the (exponentially bounded) resolvent family 
for (3) on a Banach space $X$, under what conditions does the complex inversion formula

$$
\begin{array}{r}
\lim _{N \rightarrow \infty}\left[S_{N}(t) x:=\frac{1}{2 i \pi} \int_{\alpha-i N}^{\alpha+i N} e^{\lambda t} H(\lambda) x d \lambda\right]=S(t) x \\
\left(\alpha>\omega_{0}(S), t \geq 0\right)
\end{array}
$$

holds true?

For the $C_{0}$-semigroups' case (i.e., $a(t)=1$ ) the classical result $[17$, p. 349] is that one always has the strong convergence (12) if $x \in D(A)$. Recently, the authors in [38, Proposition 4.8] have used the notion of the admissibility and proved in [38, Proposition 4.8] that this inversion takes place on larger spaces than $D(A)$. In Hilbert setting, the inversion (12) has been generalized to all $x \in X$ in [18] using Plancherel's Theorem. In the paper [19] Driouich and El-Mennaoui have extended the result in [18] in the case where $X$ has the UMD-property and it has been proved that the UMD property is essential by exhibiting an example for which the inverse Laplace transform does not always converge. This was subsequently generalized from semigroups to solution families for scalar type Volterra integral equations (3) by Cioranescu and Lizama in [22] under some regularity assumptions on the kernel $a(t)$. In particular, it has been proved in [22, Proposition 2] that for $a \in C^{1}\left(\mathbb{R}^{+}\right)$the inversion for solution families for Volterra equations (3) holds on $D(A)$. On UMD spaces, Haase in [23] has presented new and much shorter proofs of these results (under less strong assumptions on $a(t)$ ), eventually, even generalizing them. His approach uses some elementary Fourier analysis. Recently, this was generalized from resolvent families to convoluted semigroups in [24]. This inversion problem will be studied for a class of integrodifferential Volterra equations in the next section. Note that the class of integrodifferential Volterra equations has a "big" intersection with the class of Volterra integral one (partial results had been obtained earlier). Let us signalize that early, and in Hilbert setting, the first result to our knowledge on the inversion formula on $D(A)$ for scalar Volterra integrodifferential equations (3) under the conditions that $k \in C^{1}\left(\mathbb{R}^{+}\right)$and both $k$ and $k^{\prime}$ are exponentially bounded is implicitly contained in [8].

We will have the occasion to use the following observation which is the exact generalization of the corresponding wellknown case $k(t)=0$ (i.e., the semigroups) proved in [17, Theorem 11.6.1]. We sketch the proof for the reader's convenience.

Proposition 6. Let a $\in L_{\text {loc }}^{1}\left(R^{+}\right)$be exponentially bounded and let $(S(t))_{t \geq 0}$ be the exponentially bounded resolvent family for (3). Then, for each $x \in D(A)$ and $\alpha>\max \left(\omega_{0}(a), \omega_{0}(S)\right)$, we have

$$
\lim _{N \rightarrow \infty} \frac{1}{2 i \pi} \int_{\alpha-i N}^{\alpha+i N} e^{\lambda t} H(\lambda) x d \lambda= \begin{cases}S(t) x & \forall t>0 \\ \frac{x}{2} & \text { for } t=0\end{cases}
$$

in $X$, uniformly in $t$ from compact subsets of $[0, \infty[$.
Proof. Let $x \in D(A)$ and $\alpha>\max \left(w_{0}(a), w_{0}(S)\right)$ then by the resolvent equation (S3), we have $S(t) x-x=(a * S)(t) A x$, and by virtue of Proposition 5 we obtain

$$
\begin{aligned}
\widehat{(a * S)}(\lambda) A x & =\widehat{S}(\lambda) x-\widehat{1}(\lambda) x \\
& =H(\lambda) x-\frac{1}{\lambda} x
\end{aligned}
$$

for $\operatorname{Re} \lambda>\alpha$.

Thanks to [23, Proposition 2.1], for all $t \geq 0$ we have

$$
\begin{aligned}
(a * S)(t) A x & =\lim _{N \rightarrow \infty} \frac{1}{2 i \pi} \int_{\alpha-i N}^{\alpha+i N} e^{\lambda t} \widehat{(a * S)}(\lambda) A x d \lambda, \\
& =\lim _{N \rightarrow \infty} \frac{1}{2 i \pi}\left(\int_{\alpha-i N}^{\alpha+i N} e^{\lambda t} H(\lambda) x d \lambda\right. \\
& \left.-\int_{\alpha-i N}^{\alpha+i N} \frac{e^{\lambda t}}{\lambda} x d \lambda\right),
\end{aligned}
$$

where the limit exists uniformly with respect to $t$ in $[0, \infty[$.

On the other hand, we have

$$
\begin{aligned}
(a * S)(t) A x= & \lim _{N \rightarrow \infty} \frac{1}{2 i \pi} \int_{\alpha-i N}^{\alpha+i N} e^{\lambda t} H(\lambda) x d \lambda \\
& -\lim _{N \rightarrow \infty} \frac{1}{2 i \pi} \int_{\alpha-i N}^{\alpha+i N} \frac{e^{\lambda t}}{\lambda} x d \lambda .
\end{aligned}
$$

It is well-known that the second limit is $x$ if $t>0$ but $x / 2$ if $t=0$ (see, e.g., [17]).

Indeed, we known for all $x \in D(A)$ and $\alpha>w_{0}(A)$ that

$$
\lim _{N \rightarrow \infty} \frac{1}{2 i \pi} \int_{\alpha-i N}^{\alpha+i N} e^{\lambda t} R(\lambda, A) x d \lambda= \begin{cases}T(t) x & \forall t>0, \\ \frac{x}{2} & \text { for } t=0,\end{cases}
$$

in $X$, uniformly in $t$ from compact subsets of [0, $\infty$ [ where $R(\lambda, A):=(\lambda I-A)^{-1}$ is the resolvent of the semigroup $(T(t))_{t \geq 0}$.

In particular, for $T(t)=I$, the generator is $A=0$ and $R(\lambda, 0)=1 / \lambda$, thus we obtain

$$
\lim _{N \rightarrow \infty} \frac{1}{2 i \pi} \int_{\alpha-i N}^{\alpha+i N} \frac{e^{\lambda t}}{\lambda} x d \lambda= \begin{cases}x & \forall t>0, \\ \frac{x}{2} & \text { for } t=0,\end{cases}
$$

in $X$, uniformly in $t$ from compact subsets of $[0, \infty[$.

Then according to the resolvent equation (S1) and (S3) in Definition 3, for all $x \in D(A)$ and $\alpha>\max \left(w_{0}(a), w_{0}(S)\right)$ we obtain

$$
\begin{aligned}
S(t) x-x= & \lim _{N \rightarrow \infty} \frac{1}{2 i \pi} \int_{\alpha-i N}^{\alpha+i N} e^{\lambda t} H(\lambda) x d \lambda \\
& - \begin{cases}x & \forall t>0, \\
\frac{x}{2} & \text { for } t=0 .\end{cases}
\end{aligned}
$$


Hence we deduce that for all $x \in D(A)$ and $\alpha>$ $\max \left(w_{0}(a), w_{0}(S)\right)$, we have

$$
\lim _{N \rightarrow \infty} \frac{1}{2 i \pi} \int_{\alpha-i N}^{\alpha+i N} e^{\lambda t} H(\lambda) x d \lambda- \begin{cases}S(t) x & \forall t>0, \\ \frac{x}{2} & \text { for } t=0,\end{cases}
$$

in $X$, uniformly in $t$ from compact subsets of $[0, \infty[$.

\section{Integrodifferential Equation with Bounded Variation Kernels}

The purpose here is to prove some complex inversion of Laplace transform for the resolvent families of the integrodifferential Volterra equations (2). Although our hypotheses on kernel and the approach differ from the one considered by $[22,23]$, there is a big overlap in the fundamental results. For this class, it is known that the integrodifferential equation (2) can be converted to an abstract Cauchy problem on a product space (see, e.g., [15, VI.7]). This technique has been widely used (see, e.g., $[9,10,12,16,35])$. In the following, we restate some notations and related results for the sake of convenience.

This idea in the following will be applied to the case of the complex inversion formula for (2). Although it is a special case of the situation considered in the above section, it is worthwhile to deal with the partial inverse formula case first, which generalizes the result from semigroups to solution families for scalar Volterra equations (2) and constitutes an extension of a result in [8]. Next, we prove the strong convergence of the complex inversion formula for this class on UMD spaces.

It is easy to see that (2) is equivalent to

$$
x(t)=x_{0}+\int_{0}^{t}(1+1 * k)(t-s) A x(s) d s, \quad t \geq 0 .
$$

In what follows, we assume that $k \in W^{1, p}\left(\mathbb{R}^{+}\right)$and $A$ generates a $C_{0}$-semigroup $(T(t))_{t \geq 0}$ on $X$. Recall that with $k \in B V_{\text {loc }}\left(\mathbb{R}^{+}\right)$(the space of functions locally of bounded variation) only, the operator $A$ has to be a generator of $C_{0^{-}}$semigroups to obtain the well-posedness of (2), but this condition is not much restrictive, since generation of $A$ is a necessary and sufficient condition for the well-posedness of (21) (which is equivalent to (2)) (see [6, Corollary 1.4]).

From now on, we denote by $\left(S(t)_{t \geq 0}\right)$ the resolvent family associated with (21). As in [15, VI.7, Part C], we first introduce the product space $\mathscr{X}:=X \times L^{p}\left(\mathbb{R}^{+} ; X\right) 1 \leq p<\infty$, which is a Banach space with the norm

$$
\left\|\left(\begin{array}{l}
x \\
f
\end{array}\right)\right\|^{2}=\|x\|_{X}^{2}+\|f\|_{L^{p}\left(\mathbb{R}^{+}, X\right)}^{2}, \quad x \in X, f \in L^{p}\left(\mathbb{R}^{+} ; X\right) .
$$

Next, we define on $\mathscr{X}$ the operator matrices

$$
\mathscr{T}(t):=\left(\begin{array}{cc}
T(t) & R(t) \\
0 & S_{l}(t)
\end{array}\right), \quad t \geq 0
$$

where $\left(S_{l}(t)\right)_{t \geq 0}$ (the left shift semigroup) and $R(t)$ are defined on $L^{p}\left(\mathbb{R}^{+} ; X\right)$ :

$$
\begin{gathered}
\left(S_{l}(t) f\right)(\tau):=f(t+\tau), \quad \tau \geq 0 \\
R(t) f:=\int_{0}^{t} T(t-s) f(s) d s, \quad f \in L^{p}\left(\mathbb{R}^{+} ; X\right) .
\end{gathered}
$$

Then $(\mathscr{T}(t))_{t \geq 0}$ forms a $C_{0}$-semigroup on $\mathscr{X}$ with the generator given by

$$
\mathscr{A}:=\left(\begin{array}{cc}
A & \delta_{0} \\
0 & \frac{d}{d s}
\end{array}\right), \quad D(\mathscr{A}):=D(A) \times W^{1, p}\left(\mathbb{R}^{+} ; X\right),
$$

where $d / d s$ denotes the generator of the semigroup $\left(S_{l}(t)\right)_{t \geq 0}$ with domain $D(d / d s)=W^{1, p}\left(\mathbb{R}^{+} ; X\right)$, the vector-valued Sobolev space, and $\delta_{0}$ the Dirac distribution, that is, $\delta_{0}(f)=$ $f(0)$ for each $f \in W^{1, p}\left(\mathbb{R}^{+} ; X\right)$. Finally, define $M \in$ $\mathscr{L}\left(D(A) ; W^{1, p}\left(\mathbb{R}^{+} ; X\right)\right)$ as follows:

$$
\begin{aligned}
M x & :=k(\cdot) A x, \quad x \in D(A), \\
\mathscr{M}\left(\begin{array}{l}
x \\
f
\end{array}\right):= & \left(\begin{array}{cc}
0 & 0 \\
M & 0
\end{array}\right)\left(\begin{array}{l}
x \\
f
\end{array}\right)=\left(\begin{array}{c}
0 \\
M x
\end{array}\right), \\
& \left(\begin{array}{l}
x \\
f
\end{array}\right) \in D(\mathscr{M}):=D(\mathscr{A}),
\end{aligned}
$$

and denote

$$
\mathscr{A}^{V}:=\mathscr{A}+\mathscr{M}, \quad D\left(\mathscr{A}^{V}\right):=D(\mathscr{A}) .
$$

In [15, VI.7], it is shown that $\mathscr{A}^{V}$ generates a $C_{0}$-semigroup $\left(\mathscr{T}^{V}(t)\right)$ on $\mathscr{X}$. In the sequel, we denote

$$
\mathscr{T}^{V}(t)=\left(\begin{array}{ll}
T_{11}(t) & T_{12}(t) \\
T_{21}(t) & T_{22}(t)
\end{array}\right) .
$$

We now rewrite the Volterra system (2) into an equivalent Cauchy system as follows:

$$
\begin{gathered}
\dot{\mathscr{Z}}(t)=\mathscr{A}^{V} \mathscr{Z}(t), \quad t \geq 0, \\
\mathscr{Z}(0)=\mathscr{E}_{0} \in \mathscr{X} .
\end{gathered}
$$

Note that it was early observed in [6] (see also [35]) that the solutions of (21) are given by $x(t)=\pi_{1} \mathscr{T}^{V}(t) x_{0}$, where $\pi_{1}$ : $X \rightarrow X$ is the projection mapping $(x, f)$ to $x$ (see $[15$, VI.7]) for the $L^{p}$ case and [12] for $Y=C_{u b}$, the space of bounded and uniformly continuous functions. That is, $T_{11}(t)=S(t)$ for all $t \geq 0$.

Observe that the resolvent $(S(t))_{t>0}$ of $(21)$ is exponentially bounded; this extends the result in [35, when $p=2$ ] which has been obtained by a direct method. Indeed, the semigroup $\left(\mathscr{T}^{V}(t)\right)_{t \geq 0}$ is always exponentially bounded (see, e.g., [15, Proposition 5.5, p.39]); thus there exist constants $M>0$ and $\alpha \in \mathbb{R}$ such that $\left\|\mathscr{T}^{V}(t)\right\| \leq M e^{\alpha t}$. Making use of the fact that $T_{11}(t)=S(t)$, we deduce that $(S(t))_{t \geq 0}$ is also exponentially bounded and that $\omega_{0}(S) \leq \omega_{0}\left(\mathscr{A}^{V}\right)$. Thus it is permissible to consider its Laplace transform. 
By virtue of Proposition 5, the Laplace transform of $S(\cdot) x_{0}$ is well defined and it is given by

$$
\int_{0}^{\infty} e^{-\lambda t} S(t) x_{0} d t=H(\lambda) x_{0}:=(\lambda I-(1+\widehat{k}(\lambda)) A)^{-1} x_{0},
$$

for all $\operatorname{Re} \lambda>\max \left(\omega_{0}(k), \omega_{0}(S)\right)$ and $x_{0} \in X$.

The following lemma is quite useful.
Lemma 7. (i) For $\operatorname{Re} \lambda>\omega^{*}:=\max \left(0, \omega_{0}(S)\right)$ and $x \in X$, we have

$$
R\left(\lambda, \mathscr{A}^{V}\right)\left(\begin{array}{l}
x \\
0
\end{array}\right)=\left(\begin{array}{c}
H(\lambda) x \\
R\left(\lambda, \frac{d}{d s}\right) M H(\lambda) x
\end{array}\right) .
$$

(ii) For $\lambda \in \mathbb{C}_{0} \cap \rho\left(\mathscr{A}^{V}\right) \cap \rho(\mathscr{A})$, we have

$$
R\left(\lambda, \mathscr{A}^{V}\right)=\left(\begin{array}{cc}
H(\lambda) & H(\lambda) \delta_{0} R\left(\lambda, \frac{d}{d s}\right) \\
R\left(\lambda, \frac{d}{d s}\right) M H(\lambda) & R\left(\lambda, \frac{d}{d s}\right) M H(\lambda) \delta_{0} R\left(\lambda, \frac{d}{d s}\right)+R\left(\lambda, \frac{d}{d s}\right)
\end{array}\right),
$$

where $R(\lambda, d / d s)$ is the resolvent of $\left(S_{l}(t)\right)_{t \geq 0}$.

Proof. (i) Invoking (28) (see [6, page 339] for more information on $\left.\left(\mathscr{T}^{V}(t)\right)_{t \geq 0}\right)$ we have

$$
T_{21}(t)=A \int_{0}^{t} k(t-\tau+\cdot) S(\tau) d \tau \quad \forall t>0 .
$$

Thus, using (28) for all $\operatorname{Re} \lambda>\omega^{*}:=\max \left(0, \omega_{0}(S)\right)$, we have

$$
\begin{aligned}
& \int_{0}^{\infty} e^{-\lambda t} \mathscr{T}^{V}(t)\left(\begin{array}{l}
x \\
0
\end{array}\right) d t \\
& =\left(\begin{array}{c}
\int_{0}^{\infty} e^{-\lambda t} S(t) x d t \\
\int_{0}^{\infty} e^{-\lambda t}\left(A \int_{0}^{t} k(t-\tau+\cdot) S(\tau) x d \tau\right) d t
\end{array}\right) \\
& =\left(\int_{0}^{\infty} e^{-\lambda t}\left(A \int_{0}^{t} k(t-\tau+\cdot) S(\tau) x d \tau\right) d t\right) \\
& =\left(\int_{0}^{\infty} e^{-\lambda t} A \int_{0}^{t}\left(\begin{array}{c}
H(\lambda) x \\
\left(S_{l}(t-\tau) k\right)(\cdot) S(\tau) x d \tau d t
\end{array}\right)\right. \\
& =\left(\begin{array}{c}
H(\lambda) x \\
e^{\lambda \cdot} \int_{.}^{\infty} e^{-\lambda t}\left(\left(S_{l}(\cdot) M\right)(\cdot) * S\right)(t) x d t
\end{array}\right) \\
& =\left(\begin{array}{c}
H(\lambda) x \\
R\left(\lambda, \frac{d}{d s}\right) M H(\lambda) x
\end{array}\right),
\end{aligned}
$$

(ii) For each $\lambda \in \rho(A)$ with $\operatorname{Re}(\lambda)>0$, from [15, Lemmas VI.7.23-24], we know that

$$
R(\lambda, \mathscr{A})=\left(\begin{array}{cc}
R(\lambda, A) & R(\lambda, A) \delta_{0} R\left(\lambda, \frac{d}{d s}\right) \\
0 & R\left(\lambda, \frac{d}{d s}\right)
\end{array}\right)
$$

$$
I-R(\lambda, \mathscr{A}) \mathscr{M}=\left(\begin{array}{cc}
I-\widehat{k}(\lambda) R(\lambda, A) A & 0 \\
-R\left(\lambda, \frac{d}{d s}\right) M & I
\end{array}\right) .
$$

Using

$$
\begin{aligned}
\lambda I-\mathscr{A}^{V} & =\lambda I-\mathscr{A}-\mathscr{M}, \\
& =(\lambda I-\mathscr{A})(I-R(\lambda, \mathscr{A}) \mathscr{M}),
\end{aligned}
$$

we get, since $\lambda \in \rho(\mathscr{A}) \cap \rho\left(\mathscr{A}^{V}\right)$, that $[I-R(\lambda, \mathscr{A}) \mathscr{M}]$ is invertible in $\mathscr{L}(X)$ and by, for example, [31, Lemma A.4.2], we have

$$
\begin{aligned}
{[I} & -R(\lambda, \mathscr{A}) \mathscr{M}]^{-1} \\
\quad & =\left[\begin{array}{cr}
(I-\widehat{k}(\lambda) R(\lambda, A) A)^{-1} & 0 \\
R\left(\lambda, \frac{d}{d s}\right) M(I-\widehat{k}(\lambda) R(\lambda, A) A)^{-1} & I
\end{array}\right] .
\end{aligned}
$$

which implies (31).

Finally, a direct computation yields 


$$
\begin{aligned}
R\left(\lambda, \mathscr{A}^{V}\right) & =(I-R(\lambda, \mathscr{A}) \mathscr{M})^{-1} R(\lambda, \mathscr{A}) \\
& =\left(\begin{array}{cc}
H(\lambda) & H(\lambda) \delta_{0} R\left(\lambda, \frac{d}{d s}\right) \\
R\left(\lambda, \frac{d}{d s}\right) M H(\lambda) & R\left(\lambda, \frac{d}{d s}\right) M H(\lambda) \delta_{0} R\left(\lambda, \frac{d}{d s}\right)+R\left(\lambda, \frac{d}{d s}\right)
\end{array}\right) .
\end{aligned}
$$

Here is finally the result about strong convergence of the complex inversion formula.

Proposition 8. Let $(S(t))_{t \geq 0}$ be the exponentially bounded resolvent family for (2) on $X$. Suppose that $X$ is a UMD space. Then there exists $\widetilde{\omega} \geq \omega_{0}(S)$, such that for all $x \in X$ and $\alpha>\widetilde{\omega}$ one has

$$
\lim _{N \rightarrow \infty} \frac{1}{2 i \pi} \int_{\alpha-i N}^{\alpha+i N} e^{\lambda t} H(\lambda) x d \lambda=S(t) x,
$$

in $X$, uniformly in t from compact subsets of $] 0, \infty[$.

Proof. Since $X$ is a UMD, the product space $\mathscr{X}$ is a UMD (see $[45,46])$. Applying [19, Theorem 1] to the semigroup $\left(\mathscr{T}^{V}(t)\right)_{t \geq 0}$, we obtain

$$
\lim _{N \rightarrow \infty} \frac{1}{2 i \pi} \int_{\alpha-i N}^{\alpha+i N} e^{\lambda t} R\left(\lambda, \mathscr{A}^{V}\right)\left(\begin{array}{l}
x \\
f
\end{array}\right) d \lambda=\mathscr{T}^{V}(t)\left(\begin{array}{l}
x \\
f
\end{array}\right),
$$

for all $\left(\begin{array}{l}x \\ f\end{array}\right) \in \mathscr{X}$ and $\alpha>\omega_{0}\left(\mathscr{A}^{V}\right)$, where the limit exists uniformly with respect to $t$ in ] $0, \infty$ [. Taking $f=0$ in (40) and by virtue of Lemma 7(i), a straightforward computation shows that for all $\alpha>\widetilde{\omega}:=\max \left(0, \omega_{0}\left(\mathscr{A}^{V}\right)\right) \geq \omega^{*}$ we have

$$
\lim _{N \rightarrow \infty} \frac{1}{2 i \pi} \int_{\alpha-i N}^{\alpha+i N} e^{\lambda t} H(\lambda) x d \lambda=S(t) x,
$$

for all $x \in X$, uniformly for $t \in] 0, \infty[$.

We see that the results in [22, Theorem 1], [23, Theorem 4.2], and Proposition 8 assert only strong convergence and uniformity in $t$ from compact subsets of ]0, $\infty[$. As for the semigroups' case, it would be of interest to see whether, for scalar Volterra integral systems, this convergence holds true for $t=0$ (it does not for $x \in D(A)$, (see Proposition 6)). For the semigroups, which are the special cases of scalar Volterra integral equations, an affirmative answer was given in [38, Proposition 2.3].

The next result is a version of [38, Proposition 2.3] for resolvent family.

Proposition 9. Let $a \in L_{\mathrm{loc}}^{1}\left(R^{+}\right)$be exponentially bounded and let $(S(t))_{t \geq 0}$ be the exponentially bounded resolvent family for (3). If A generates an analytic $C_{0}$-semigroup in $X$, then for all $x \in X$, and $\alpha>\max \left(\omega_{0}(S), \omega_{0}(a)\right)$ the integral $\int_{\alpha-i N}^{\alpha+i N} H(\lambda) x d \lambda$ converges in $X$ as $N \rightarrow \infty$, hence, in $D(A)$ for all $x \in D(A)$.
Proof. The proof is more or less the same for the semigroups' case. Assume first that $x \in D(A)$. Then for all $0 \neq \lambda>\omega$ and using the resolvent identity we obtain

$$
H(\lambda) x=\frac{x}{\lambda}+\widehat{a}(\lambda) H(\lambda) A x .
$$

Let $\partial \mathscr{C}_{N}^{+}$be the boundary of the half-disc $\mathscr{C}_{N}^{+}$defined by $\mathscr{C}_{N}^{+}=\{\lambda \in \mathbb{C}:|\lambda| \leq N,|\arg \lambda| \leq \pi / 2\}$. Then Cauchy's theorem yields

$$
\begin{aligned}
\int_{\alpha-i N}^{\alpha+i N} H(\lambda) x d \lambda & =-i \int_{\partial \mathscr{C}_{N}^{+}} H(\lambda) x d z \\
& =\int_{-(\pi / 2)}^{\pi / 2} N e^{i \theta} H\left(N e^{i \theta}\right) x d \theta .
\end{aligned}
$$

Since $A$ generates an analytic semigroup on $X$, the set $\left\{\left(1 / \widehat{a}\left(N e^{i \theta}\right)\right) R\left(\left(1 / \widehat{a}\left(N e^{i \theta}\right)\right), A\right)\right\}$ is uniformly bounded in $\mathscr{C}_{N}^{+}$ (see [47]) and, hence, $\left\{N e^{i \theta} H\left(N e^{i \theta}\right)\right\}$ is uniformly bounded in $\mathscr{C}_{N}^{+}$. It follows that $\int_{\alpha-i N}^{\alpha+i N} H(\lambda) x d \lambda$ is uniformly bounded in $\mathscr{C}_{N}^{+}$. Using once again the resolvent identity we obtain

$$
\begin{aligned}
& \int_{\partial \mathscr{C}_{N}^{+}} H(\lambda) x d z \\
& \quad=\int_{\partial \mathscr{C}_{N}^{+}} \frac{d \lambda}{\lambda} x+\int_{\partial \mathscr{C}_{N}^{+}} \widehat{a}(\lambda) H(\lambda) A x d \lambda \\
& =\pi i x+i \int_{-(\pi / 2)}^{\pi / 2} \widehat{a}\left(N e^{i \theta}\right) N e^{i \theta} H\left(N e^{i \theta}\right) A x d \theta,
\end{aligned}
$$

for all $x \in D(A)$. By the fact that $A$ generates an analytic semigroup and by virtue of Riemann-Lebesgue's theorem (see [20, Theorem 1.8.1.c]), the integral on the right-hand side of the above equality converges to zero as $N \rightarrow \infty$. Using the last equality, the density of $D(A)$ in $X$, and the fact that the integral $\int_{\alpha-i N}^{\alpha+i N} H(\lambda) x d \lambda$ is uniformly bounded in $\mathscr{C}_{N}^{+}$, an elementary equicontinuity argument guarantees the convergence in $X$ for every $x \in X$ thanks to Proposition 6 . Now, let $x \in D(A)$ and $y_{0}:=\left(\lambda_{0} I-A\right) x$ for some $\lambda_{0} \in \rho(A)$. Then we get

$$
\int_{\alpha-i N}^{\alpha+i N} H(\lambda) x d \lambda=R\left(\lambda_{0}, A\right) \int_{\alpha-i N}^{\alpha+i N} H(\lambda) y_{0} d \lambda .
$$

Since $A R\left(\lambda_{0}, A\right) \in \mathscr{L}(X)$, we conclude that $\int_{\alpha-i N}^{\alpha+i N} H(\lambda) x d \lambda$ converges in $D(A)$. 
Finally, combining Propositions 8 and 9 leads to the following corollary.

Corollary 10. Let $A$ be the generator of an analytic $C_{0^{-}}$ semigroup in $X$ and let $(S(t))_{t \geq 0}$ be the resolvent family for (2). Suppose that $X$ is a UMD space. Then, for all $x \in X$ (resp., $x \in D(A))$ and $\alpha>\omega^{*}$, one has

$$
\lim _{N \rightarrow \infty} \frac{1}{2 i \pi} \int_{\alpha-i N}^{\alpha+i N} e^{\lambda t} H(\lambda) x d \lambda= \begin{cases}S(t) x & \forall t>0 \\ \frac{x}{2} & \text { for } t=0\end{cases}
$$

in $X$ (resp., in $D(A)$ ), which is uniform on $t$ for any compact interval of $[0, \infty[$.

\section{Characterization of Admissibility}

We now turn our attention back to the notion of admissibility. In this section we present sufficient and necessary conditions for the $L^{p}$-admissibility of control operators for integrodifferential Volterra control systems (1) which has the following equivalent form:

$$
\begin{gathered}
x(t)=x_{0}+\int_{0}^{t}(1+1 * k)(t-s) A x(s) d s \\
+\int_{0}^{t} B u(s) d s, \quad t \geq 0, \\
x(0)=x_{0} \in X,
\end{gathered}
$$

extending the result for the semigroups [38, Proposition 3.2]. Here we assume that the control operator $B \in \mathscr{L}\left(U ; X_{-1}\right)$ where $X_{-1}$ is the extrapolation space with respect to $A$ (see, e.g., [15]) and $U$ is another Banach space. It is further assumed that $k \in L_{\text {loc }}^{1}\left(\mathbb{R}^{+}\right)$is exponentially bounded and that the uncontrolled system (i.e., (21)) admits an exponentially bounded resolvent family $(S(t))_{t \geq 0}$.

Since the resolvent of (21) commutes with the operator $A$, then it can be easily seen that the restriction $\left(S_{1}(t)\right)_{t \geq 0}$ to $X_{1}$ of $(S(t))_{t \geq 0}$, the solution of (21), is strongly continuous. Moreover, since $\rho(A) \neq \varnothing$ (see Proposition 5) $\left(S_{1}(t)\right)_{t \geq 0}$ solves for each $x_{0} \in X$ and $A_{1}$ replacing $A$. Likewise, $S(t)$ has a unique bounded extension to $X_{-1}$ for each $t \geq 0$ and $t \mapsto$ $S_{-1}(t)$ is also strongly continuous, and it solves $(21)$ in $X_{-1}$ with $A_{-1}$ replacing $A$.

The mild solution of (47) is formally given by the variation of the constant formula

$$
x(t)=S(t) x_{0}+\int_{0}^{t} S_{-1}(t-s) B u(s) d s,
$$

which is actually the classical solution if $B \in \mathscr{L}(U, X), x_{0} \in$ $D(A)$, and $u$ is sufficiently smooth. In general however, $B$ is not a bounded operator from $U$ into $X$ and so an additional assumption on $B$ will be needed to ensure that $x(t) \in X$ for every $x_{0} \in X$ and every $u \in L^{p}\left(\mathbb{R}^{+} ; U\right)$ or $L_{\text {loc }}^{p}\left(\mathbb{R}^{+} ; U\right)$.

In the same spirit of the semigroups' case, the following are the most natural definitions of the $L^{p}$-admissibility for resolvent families.
Definition 11. Let $B \in \mathscr{L}\left(U ; X_{-1}\right)$ and $p \in[1, \infty[$.

(i) $B$ is called (infinite-time) an $L^{p}$-admissible operator for $\left(S(t)_{t \geq 0}\right)$, if there exists a constant $M>0$, such that

$$
\begin{array}{r}
\left\|S_{-1} * B u(t)\right\|_{X} \leq M\|u\|_{L^{p}([0, \infty[; U)} \\
\forall u \in L^{p}([0, \infty[; U), t>0 .
\end{array}
$$

(ii) $B$ is called a finite-time $L^{p}$-admissible operator for $(S(t))_{t \geq 0}$ if there exist $t_{0}>0$ and a constant $M\left(t_{0}\right)>0$, such that

$$
\begin{array}{r}
\left\|S_{-1} * B u\left(t_{0}\right)\right\|_{X} \leq M\left(t_{0}\right)\|u\|_{L^{p}\left(\left[0, t_{0}\right] ; U\right)} \\
\forall u \in L^{p}\left(\left[0, t_{0}\right] ; U\right) .
\end{array}
$$

Note that the definition of (infinite-time) $L^{p}$-admissible control operator for $\left(S(t)_{t \geq 0}\right)$ was introduced in [34] when $p=2$ and implies the finite-time $L^{2}$-admissibility condition considered in [33]. Our definitions of finite- and infinitetime $L^{p}$-admissible control operator for $\left(S(t)_{t \geq 0}\right)$ correspond to that of the semigroups and also imply that of [33] when $p=2$. It is well known that $\left(P_{1}\right)$ : finite-time $L^{p}$-admissibility and the uniform finite-time $L^{p}$-admissibility, which means that for all $t>0$ there exists a constant $M(t)>0$, such that $\left\|S_{-1} * B u(t)\right\|_{X} \leq M(t)\|u\|_{L^{p}([0, t] ; U)}$ for all $u \in L^{p}([0, t] ; U)$, are equivalent for semigroups and $\left(P_{2}\right)$ : finite-time $L^{P_{-}}$ admissibility and the infinite-time $L^{p}$-admissibility are equivalent for exponentially stable semigroups. We emphasize that, in [35], the authors have found an example (i.e., (2) with $A$ generator of exponentially stable semigroup) for which finitetime $L^{2}$-admissibility and infinite-time $L^{2}$-admissibility are not equivalent, but, in their example, we can see that the associated resolvent family is not exponentially stable. Thus, a question that remains open to our knowledge is, whether for Volterra integral systems, these problems (i.e., $\left(P_{1}\right)-\left(P_{2}\right)$ ) are still true for resolvent families. In [37, Corollary 5.4 and Proposition 5.6], partial answers were given to these problems when $p=1$.

It has been observed in [36, for $p=2$ ] (resp., [37, for $p \geq 1]$ ) that $L^{2}$ - (resp., $\left.L^{p}\right)$ admissibility of $B \in \mathscr{L}\left(U ; X_{-1}\right)$ is equivalent to the fact that there exists a constant $M>0$, such that

$$
\left\|\int_{0}^{\infty} S_{-1}(t) B u(t) d t\right\|_{X} \leq M\|u\|_{L^{p}\left(\mathbb{R}^{+} ; U\right)},
$$

for all $u \in L_{c}^{p}\left(\mathbb{R}^{+} ; U\right)$ (the space of functions in $L^{p}\left(\mathbb{R}^{+} ; U\right)$ with compact support).

Of course, $L^{p}$-admissibility of $B$ guarantees that the operator $\mathfrak{B}_{\infty}: L_{c}^{p}\left(\mathbb{R}^{+} ; U\right) \rightarrow X$, given by

$$
\mathfrak{B}_{\infty} u:=\int_{0}^{\infty} S_{-1}(t) B u(t) d t
$$

possesses an extension to a linear bounded operator from $L^{p}\left(\mathbb{R}^{+} ; U\right)$ to $X$. We denote this extension again by $\mathfrak{B}_{\infty}$.

As for $p=2$ (see [36]), it is easy to verify that under $\omega_{0}(S)<0$ formula (52) holds for every $u \in L^{2}\left(\mathbb{R}^{+} ; U\right)$. 
Thanks to Proposition 5, the Laplace-transform of $S_{-1}(\cdot)$ is well defined and it is given by

$$
\begin{array}{r}
\widehat{S_{-1}}(\lambda)=\left(\lambda I-(1+\widehat{k}(\lambda)) A_{-1}\right)^{-1}=: H_{-1}(\lambda) \\
\forall \operatorname{Re}(\lambda)>\max \left(\omega_{0}(k), \omega_{0}(S)\right) .
\end{array}
$$

In the sequel we use the following notations.

For $N \geq 0, B \in \mathscr{L}\left(U ; X_{-1}\right), u \in L^{p}([0, \tau] ; U)$, and $\tau \in$ $[0, \infty]$ we set

$$
\begin{aligned}
\varphi_{N}^{\tau}(u) & =\int_{0}^{\tau} S_{-N}(\sigma) B u(\sigma) d \sigma, & \varphi_{N}(u):=\varphi_{N}^{\infty}(u), \\
\varphi^{\tau}(u) & =\int_{0}^{\tau} S_{-1}(\sigma) B u(\sigma) d \sigma, & \varphi(u):=\varphi^{\infty}(u),
\end{aligned}
$$

with

$$
S_{-N}(t) x:=\frac{1}{2 i \pi} \int_{\alpha-i N}^{\alpha+i N} e^{\lambda t} H_{-1}(\lambda) x d \lambda \quad \forall x \in X_{-1} .
$$

We may now formulate and prove the main result of this section by giving a necessary or/and sufficient condition for finite- (or infinite-) time $L^{p}$-admissibility of $B$. The necessary condition here is essentially based on a geometric property of the underlying control space $U$ that is the UMDproperty. The result encompasses Hilbert control spaces, but the proposition below yields the criterion's necessity.

Theorem 12. Let $B \in \mathscr{L}\left(U ; X_{-1}\right)$. Then the following assertions hold.

(i) If $\varphi_{N}^{\tau_{0}}$ is uniformly bounded on $\mathscr{L}\left(L^{p}\left(\left[0, \tau_{0}\right] ; U\right) ; X\right)$ for some $\tau_{0}>0$ then $B$ is finite-time $L^{p}$-admissible for $(S(t))_{t \geq 0}$.

(ii) Assume that $U$ is a UMD space, $p>1$, and $\omega_{0}(S)<0$. Then $B$ is $L^{p}$-admissible for $(S(t))_{t \geq 0}$ if and only if $\varphi_{N}$ is uniformly bounded on $\mathscr{L}\left(L_{c}^{p}\left(\mathbb{R}^{+} ; U\right) ; X\right)$.

Proof. Part (i). The proof of this is similar to that of [38, Proposition 3.2]. Notice that, for any $\mu \in \rho(A)$ and constant input $u_{0} \in U$, we have $\mu^{2} R^{2}(\mu, A) B u_{0} \in D(A)$. By Proposition 6, we have

$$
X-\lim _{N \rightarrow \infty} S_{N}\left(\tau_{0}\right) \mu^{2} R^{2}(\mu, A) B u_{0}=S\left(\tau_{0}\right) \mu^{2} R^{2}(\mu, A) B u_{0} .
$$

Thanks to [48, Lemma 1] the integral $\int_{\varepsilon}^{\tau_{0}} S_{-1}(\tau) B u(\tau) d \tau$ takes value in $X$ due to the fact that the considered input $u(t)$ is a step function. Thus by a density argument, to prove that $B$ is finite-time $L^{p}$-admissible (for time $\tau_{0}$ ) for $(S(t))_{t \geq 0}$, it suffices to prove that for any step function $u:\left[0, \tau_{0}\right] \rightarrow U$ with compact support that does not contain zero, the following uniform estimate

$$
\left\|\int_{0}^{\tau_{0}} S_{-1}(\tau) B u(\tau) d \tau\right\|_{X} \leq M_{\tau_{0}}\|u\|_{L^{p}\left(\left[0, \tau_{0}\right] ; U\right)},
$$

holds for some $M_{\tau_{0}}>0$.
So, consider a step function $u$ with compact support that does not contain zero. Then there exists $\varepsilon>0$ such that

$$
\begin{aligned}
\varphi_{N}^{\tau_{0}}(u) & :=\int_{0}^{\tau_{0}} S_{-N}(\sigma) B u(\sigma) d \sigma, \\
& =\int_{\varepsilon}^{\tau_{0}} S_{-N}(\sigma) B u(\sigma) d \sigma .
\end{aligned}
$$

Since $\left(\varphi_{N}^{\tau_{0}}\right)_{N}$ is uniformly bounded with $\left\|\varphi_{N}^{\tau_{0}}\right\| \leq K_{\tau_{0}}$ for some $K_{\tau_{0}}>0$ and for all $N>0$, then $\mu^{2} R^{2}(\mu, A) \varphi_{N}^{\tau_{0}}$ is uniformly bounded as $\mu \rightarrow \infty$ (see [48, Corollary 3]) and we have for some $K, \mu_{0}>0$ and for all $\mu>\mu_{0}$

$$
\begin{aligned}
\left\|\mu^{2} R^{2}(\mu, A) \varphi_{N}^{\tau_{0}}(u)\right\|_{X} & =\left\|\int_{\varepsilon}^{\tau_{0}} S_{N}(\sigma) \mu^{2} R^{2}(\mu, A) B u(\sigma) d \sigma\right\|_{X} \\
& \leq K_{\tau_{0}} K\|u\|_{L^{p}\left(\left[0, \tau_{0}\right] ; U\right)} .
\end{aligned}
$$

Invoking (56), an elementary equicontinuity argument, and vector-valued dominated convergence theorem in $X$, respectively, we obtain

$$
\begin{gathered}
X-\lim _{N \rightarrow \infty} \int_{\varepsilon}^{\tau_{0}} S_{N}(\sigma) \mu^{2} R^{2}(\mu, A) B u(\sigma) d \sigma \\
=\int_{\varepsilon}^{\tau_{0}} S(\sigma) \mu^{2} R^{2}(\mu, A) B u(\sigma) d \sigma,
\end{gathered}
$$

which implies via (59) that for all $\mu>\mu_{0}$

$$
\begin{aligned}
& \left\|\int_{\varepsilon}^{\tau_{0}} S(\sigma) \mu^{2} R^{2}(\mu, A) B u(\sigma) d \sigma\right\|_{X} \\
& \quad \leq \sup _{N \geq 0}\left\|\int_{\varepsilon}^{\tau_{0}} S_{N}(\sigma) \mu^{2} R^{2}(\mu, A) B u(\sigma) d \tau\right\|_{X}, \\
& \quad \leq K_{\tau_{0}} K\|u\|_{L^{p}\left(\left[0, \tau_{0}\right] ; U\right) .}
\end{aligned}
$$

In other words, we have

$$
\begin{aligned}
& \left\|\int_{\varepsilon}^{\tau_{0}} S(\sigma) \mu^{2} R^{2}(\mu, A) B u(\sigma) d \sigma\right\|_{X} \\
& \quad=\left\|\mu^{2} R^{2}(\mu, A) \int_{\varepsilon}^{\tau_{0}} S_{-1}(\sigma) B u(\sigma) d \sigma\right\|_{X},
\end{aligned}
$$


due to the fact that $S(t)$ commute with $R(\mu, A)$ (see [48, Theorem 7]).

Passing to the limit (i.e., $\mu \rightarrow \infty$ ) in the above inequality, we deduce

$$
\left\|\int_{\mathcal{\varepsilon}}^{\tau_{0}} S_{-1}(\sigma) B u(\sigma) d\right\|_{X} \leq K_{\tau_{0}} K\|u\|_{L^{p}\left(\left[0, \tau_{0}\right] ; U\right)} .
$$

The same argument also shows that

$$
\left\|\int_{\varepsilon}^{\varepsilon^{\prime}} S_{-1}(\sigma) B u(\sigma) d \sigma\right\|_{X} \leq K_{\tau_{0}} K\left\|u \chi_{\left[\varepsilon, \varepsilon^{\prime}\right]}\right\|_{L^{p}\left(\left[0, \tau_{0}\right] ; U\right)}
$$

which implies that the sequence $\left(\int_{\varepsilon}^{\tau_{0}} S_{-1}(\sigma) B u(\sigma) d \sigma\right)_{\varepsilon}$ is a Cauchy sequence in $X$. Moreover,

$X_{-1}-\lim _{\varepsilon \rightarrow 0} \int_{\varepsilon}^{\tau_{0}} S_{-1}(\sigma) B u(\sigma) d \sigma=\int_{0}^{\tau_{0}} S_{-1}(\sigma) B u(\sigma) d \sigma \in X$.

By virtue of $X \hookrightarrow X_{-1}$, and the fact that $\int_{\mathcal{E}}^{\tau_{0}} S_{-1}(\sigma) B u(\sigma) d \sigma$ satisfies (63), we find

$$
\left\|\int_{0}^{\tau_{0}} S_{-1}(\sigma) B u(\sigma) d \sigma\right\|_{X} \leq M\left(\tau_{0}\right)\|u\|_{L^{p}\left(\left[0, \tau_{0}\right] ; U\right)},
$$

which completes the proof of (i).

Part (ii): $(\Rightarrow)$ Assume that $B$ is $L^{p}$-admissible for $(S(t))_{t \geq 0}$. Let $u \in L_{c}^{p}\left(\mathbb{R}^{+} ; U\right)$, with $\operatorname{Supp}(u)=[a, b]$. By Fubini's theorem we have

$$
\begin{aligned}
\varphi_{N}(u) \\
=\int_{a}^{b} S_{-N}(\sigma) B u(\sigma) d \sigma \\
=\frac{1}{2 \pi} \int_{a}^{b} \int_{-N}^{N} e^{(\alpha+i \lambda) \sigma} H_{-1}(\alpha+i \lambda) B u(\sigma) d \lambda d \sigma \\
=\frac{1}{2 \pi} \int_{-N}^{+N} \int_{a}^{b} e^{(\alpha+i \lambda) \sigma} H_{-1}(\alpha+i \lambda) B u(\sigma) d \sigma d \lambda \\
=\frac{1}{2 \pi} \int_{-N}^{+N} \int_{a}^{b} \int_{0}^{\infty} e^{-(\alpha+i \lambda) t} S_{-1}(t) e^{(\alpha+i \lambda) \sigma} B u(\sigma) d t d \sigma d \lambda \\
=\frac{1}{2 \pi} \int_{a}^{b} \int_{0}^{\infty} \int_{-N}^{N} e^{(\alpha+i \lambda)(\sigma-t)} S_{-1}(t) B u(\sigma) d \lambda d t d \sigma \\
=\frac{1}{2 \pi} \int_{a}^{b} \int_{0}^{\infty}\left[\int_{-N}^{+N} e^{(\alpha+i \lambda)(\sigma-t)} d \lambda\right] S_{-1}(t) B u(\sigma) d t d \sigma
\end{aligned}
$$

$$
\begin{aligned}
& =\frac{1}{2 i \pi} \int_{0}^{\infty} e^{-\alpha t} \\
& \quad \cdot \int_{a}^{b} e^{\alpha \sigma} \cdot \frac{e^{i N(\sigma-t)}-e^{-i N(\sigma-t)}}{t-\sigma} S_{-1}(t) B u(\sigma) d \sigma d t \\
& =\int_{0}^{\infty} S_{-1}(t) B e^{-\alpha t} \\
& \quad \times \int_{\mathbb{R}^{+}} e^{\alpha \sigma} \cdot \frac{\sin N(\sigma-t)}{\pi(\sigma-t)} \chi_{[a, b]} u(\sigma)(\sigma) d \sigma d t, \\
& =\int_{0}^{\infty} S_{-1}(t) B e^{-\alpha t} u_{N}^{\alpha}(t) d t .
\end{aligned}
$$

The use of Fubini theorem in this chain of equalities is justified by the fact that the maps $(\sigma, \lambda) \rightarrow e^{\lambda \sigma} H_{-1}(\lambda) B u(\sigma)$ and $(t, \sigma, \lambda) \rightarrow e^{\lambda(\sigma-t)} S_{-1}(t) B u(\sigma)$ belong to $L^{1}([a, b] \times[\alpha-$ $i N, \alpha+i N] ; X)$, and to $L^{1}\left(\mathbb{R}^{+} \times[a, b] \times[\alpha-i N, \alpha+i N] ; X_{-1}\right)$, respectively.

Here the new input $u_{N}^{\alpha}$ is given by

$$
\begin{aligned}
& u_{N}^{\alpha}(t) \\
& :=\int_{0}^{\infty} \frac{\sin N(\sigma-t)}{\pi(\sigma-t)}\left(e^{\alpha \cdot} \chi_{[a, b]}\right)(\sigma) u(\sigma) d \sigma \\
& =\left(D_{N} * f_{\alpha}\right)(t), \quad \text { with } f_{\alpha}(\cdot)=e^{\alpha \cdot} \chi_{[a, b]}(\cdot) u \in L^{p}\left(\mathbb{R}^{+} ; U\right),
\end{aligned}
$$

and $D_{N}$ denotes the Dirichlet kernel given by

$$
D_{N}(t)=\frac{\sin (N t)}{\pi t} \quad(t \in \mathbb{R}) .
$$

Following the lines of the proof of [23, Lemma 3.2], one establishes that for a UMD space $Z$ and $p \in] 1, \infty[$ we have

$$
\begin{aligned}
& D_{N} * g \longrightarrow g \quad \text { in } L^{p}\left(\mathbb{R}^{+} ; Z\right) \\
& \text { as } N \longrightarrow \infty \quad \forall g \in L^{p}\left(\mathbb{R}^{+} ; Z\right) .
\end{aligned}
$$

Thus, making use of the fact that $U$ is a UMD space, appealing (70), we obtain

$$
D_{N} * f_{\alpha} \longrightarrow f_{\alpha} \quad \text { in } L^{p}\left(\mathbb{R}^{+} ; U\right), \quad \text { as } N \longrightarrow \infty .
$$

Then, using the $L^{p}$-admissibility of $B$ and the fact that $\omega_{0}(S)<$ 0 , we obtain

$$
\begin{aligned}
& \left\|\varphi_{N_{1}}(u)-\varphi_{N_{2}}(u)\right\|_{X} \\
& =\left\|\int_{0}^{\infty} S_{-1}(t) B e^{-\alpha t}\left[u_{N_{1}}^{\alpha}(t)-u_{N_{2}}^{\alpha}(t)\right] d t\right\|_{X} \\
& =\left\|\mathfrak{B}_{\infty}\left(e^{-\alpha \cdot}\left(u_{N_{1}}^{\alpha}-u_{N_{2}}^{\alpha}\right)\right)\right\|_{X} \\
& \quad \leq M\left\|u_{N_{1}}^{\alpha}-u_{N_{2}}^{\alpha}\right\|_{L^{p}\left(\mathbb{R}^{+} ; U\right)},
\end{aligned}
$$

for $N_{1}>N_{2}>0$, which implies that $\left(\varphi_{N}(u)\right)_{N>0}$ is a Cauchy sequence in $X$. Thus $\left(\varphi_{N}(u)\right)_{N \geq 0}$ converges in $X$ as $N \rightarrow \infty$, 
for all $u \in L_{c}^{p}\left(\mathbb{R}^{+} ; U\right)$ and that $\left(\varphi_{N}\right)_{N \geq 0}$ is uniformly bounded in $\mathscr{L}\left(L_{c}^{p}\left(\mathbb{R}^{+} ; U\right), X\right)$ according to Banach-Steinhaus's theorem. Furthermore, using once again $e^{-\alpha^{*}} u_{N}^{\alpha} \rightarrow e^{-\alpha .} f_{\alpha}=u$ in $L^{p}\left(\mathbb{R}^{+} ; U\right)$, we obtain $\int_{0}^{\infty} S_{-1}(t) B e^{-\alpha t} u_{N}^{\alpha}(t) d t$ converges to $\int_{0}^{\infty} S_{-1}(t) B u(t) d t(=\varphi(u))$ in $X_{-1}$ as $N \rightarrow \infty$. By virtue of $X \hookrightarrow X_{-1}$ with continuous injection and the fact that $\left(\varphi_{N}(u)\right)_{N \geq 0}$ converges in $X$ as $N \rightarrow \infty$, we deduce that $\varphi_{N}(u)$ converges to $\varphi(u)$ in $X$ as $N \rightarrow \infty$, for all $u \in$ $L_{c}^{p}\left(\mathbb{R}^{+} ; U\right)$.

$(\Leftarrow)$ Assume that $\varphi_{N}$ is uniformly bounded on $\mathscr{L}\left(L_{c}^{p}\left(\mathbb{R}^{+} ; U\right) ; X\right)$. For $\tau_{0}>0$ and $u \in L^{p}\left(\mathbb{R}^{+} ; U\right)$ we have $v:=u \cdot \chi_{\left[0, \tau_{0}\right]} \in L_{c}^{p}\left(\mathbb{R}^{+} ; U\right)$ and

$$
\left\|\varphi_{N}(v)\right\|_{X}=\left\|\varphi_{N}^{\tau_{0}}(u)\right\|_{X} \leq \kappa\|u\|_{L^{p}\left(\left[0, \tau_{0}\right] ; U\right)}
$$

for some $\kappa>0$.

Thanks to (i) we deduce that $B$ is finite-time $L^{P_{-}}$ admissible (for time $\tau_{0}$ ) for $(S(t))_{t>0}$. By examining the proof of (i) we deduce that $B$ is $L^{p}$-admissible for $(S(t))_{t \geq 0}$. This ends the proof.

Remark 13. (1) The result of Theorem 12 may extend to systems of Volterra integral equations (3) provided that $\int_{0}^{t} S(\sigma) x d \sigma$ takes value in $D(A)$ for all $x \in X$. One may of course ask whether it is a severe restriction to consider only $a(t)=1+(1 * k)(t)$. This will be a subject of a forthcoming work.

(2) We can retrieve the result stated in Proposition 8 by using [38, Remark 3.5 (ii)]. Indeed, let $\mathscr{B}=I d_{\mathscr{X}}$, then $\mathscr{B}$ is infinite-time $L^{p}$-admissible for $\left(\mathscr{T}^{V, \omega}(t):=e^{-\omega t} \mathscr{T}^{V}(t)\right)_{t \geq 0}$ with $\omega>\omega_{0}\left(\mathscr{A}^{V}\right)$. In fact, for $t_{0}>0$ and $x \in X$, consider the input $u_{0}(t):=\mathscr{T}^{V, \omega}\left(t_{0}-t\right)\left(\begin{array}{c}x \\ 0\end{array}\right) \chi_{\left[t_{0} / 2, t_{0}\right]}$, we set:

$$
\Phi_{N}\left(u_{0}\right):=\int_{0}^{\infty} \mathscr{T}_{N}^{V, \omega}(\sigma) u_{0}(\sigma) d \sigma
$$

with

$$
\mathscr{T}_{N}^{V, \omega}(t)\left(\begin{array}{l}
x \\
f
\end{array}\right):=\frac{1}{2 \pi} \int_{-N}^{N} e^{i \lambda t} R\left(\omega+i \lambda, \mathscr{A}^{V}\right)\left(\begin{array}{l}
x \\
f
\end{array}\right) d \lambda
$$

Thanks to [38, Remark 3.5] we have

$$
\Phi_{N}\left(u_{0}\right)=\frac{t_{0}}{2} \mathscr{T}_{N}^{V, \omega}\left(t_{0}\right)\left(\begin{array}{l}
x \\
0
\end{array}\right)+L_{N}^{\omega}\left(t_{0}\right)\left(\begin{array}{l}
x \\
0
\end{array}\right)
$$

with $L_{N}^{\omega}\left(t_{0}\right) \in \mathscr{L}(\mathscr{X})$ and $L_{N}^{\omega}\left(t_{0}\right)\left(\begin{array}{l}x \\ 0\end{array}\right) \rightarrow 0$ as $N \rightarrow \infty$ for all $x \in X$.

Passing to the limit in (76) as $N \rightarrow \infty$ and invoking [38, Corollary 4.2] we obtain

$$
\Phi_{N}\left(u_{0}\right) \longrightarrow \Phi\left(u_{0}\right) \quad \text { as } N \longrightarrow \infty \text { in } \mathscr{X}
$$

with

$$
\begin{aligned}
& \Phi\left(u_{0}\right):=\int_{0}^{\infty} \mathscr{T}^{V, \omega}(\sigma) u_{0}(\sigma) d \sigma \\
& =\int_{0}^{\infty} \mathscr{T}^{V, \omega}(\sigma) \mathscr{T}^{V, \omega}\left(t_{0}-\sigma\right)\left(\begin{array}{l}
x \\
0
\end{array}\right) \chi_{\left[t_{0} / 2, t_{0}\right]} d \sigma \\
& =\int_{t_{0} / 2}^{t_{0}} \mathscr{T}^{V, \omega}\left(t_{0}\right)\left(\begin{array}{l}
x \\
0
\end{array}\right) d \sigma \\
& =\frac{t_{0}}{2} \mathscr{T}^{V, \omega}\left(t_{0}\right)\left(\begin{array}{l}
x \\
0
\end{array}\right) \\
& =\frac{t_{0}}{2} \cdot e^{-\omega t_{0}} \mathscr{T}^{V}\left(t_{0}\right)\left(\begin{array}{l}
x \\
0
\end{array}\right) \\
& =\frac{t_{0}}{2} \cdot e^{-\omega t_{0}}\left(\begin{array}{cc}
S\left(t_{0}\right) & T_{12}\left(t_{0}\right) \\
T_{21}\left(t_{0}\right) & T_{22}\left(t_{0}\right)
\end{array}\right)\left(\begin{array}{l}
x \\
0
\end{array}\right) \\
& =\frac{t_{0}}{2} \cdot e^{-\omega t_{0}}\left(\begin{array}{c}
S\left(t_{0}\right) x \\
T_{21}\left(t_{0}\right) x
\end{array}\right) .
\end{aligned}
$$

In other words, for $\omega>\omega^{*}\left(>\omega_{0}\left(\mathscr{A}^{V}\right)\right)$ we have

$$
\begin{aligned}
\mathscr{T}_{N}^{V, \omega}\left(t_{0}\right)\left(\begin{array}{l}
x \\
0
\end{array}\right) & =\frac{1}{2 \pi} \int_{-N}^{N} e^{i \lambda t_{0}} R\left(\omega+i \lambda, \mathscr{A}^{V}\right)\left(\begin{array}{l}
x \\
0
\end{array}\right) d \lambda \\
& =\frac{1}{2 i \pi} \int_{\omega-i N}^{\omega+i N} e^{(\mu-\omega) t_{0}} R\left(\mu, \mathscr{A}^{V}\right)\left(\begin{array}{l}
x \\
0
\end{array}\right) d \mu \\
& =\frac{1}{2 i \pi} \int_{\omega-i N}^{\omega+i N} e^{-\omega t_{0}} \cdot e^{\mu t} R\left(\mu, \mathscr{A}^{V}\right)\left(\begin{array}{l}
x \\
0
\end{array}\right) d \mu .
\end{aligned}
$$

Using Lemma 7(i) we obtain

$$
\begin{aligned}
& \mathscr{T}_{N}^{V, \omega}\left(t_{0}\right)\left(\begin{array}{l}
x \\
0
\end{array}\right) \\
& =\frac{1}{2 i \pi} \int_{\omega-i N}^{\omega+i N} e^{-\omega t_{0}} \cdot\left(R\left(\mu, \frac{d}{d s}\right) M H(\mu) x\right) d \mu \\
& =\left(\begin{array}{c}
e^{-\omega t_{0}} S_{N}\left(t_{0}\right) x \\
\frac{1}{2 i \pi} \int_{-N}^{N} e^{i \mu t_{0}} R\left(\omega+i \mu, \frac{d}{d s}\right) M H(\omega+i \mu) x d \mu
\end{array}\right) .
\end{aligned}
$$

Combining (76), (77), and (80), we deduce that

$$
\begin{aligned}
\frac{t_{0}}{2} \cdot e^{-\omega t_{0}} S_{N}\left(t_{0}\right) x & \longrightarrow \frac{t_{0}}{2} \cdot e^{-\omega t_{0}} S\left(t_{0}\right) x \\
\text { as } N & \longrightarrow \infty \forall x \in X,
\end{aligned}
$$

which in turns implies that

$$
\begin{array}{r}
\lim _{N \rightarrow \infty} \frac{1}{2 i \pi} \int_{\omega-i N}^{\omega+i N} e^{\lambda t} H(\lambda) x d \lambda=S(t) x \\
\text { as } N \longrightarrow \infty \forall x \in X,
\end{array}
$$

uniformly in $t$ from compact subsets of $] 0, \infty[$. 


\section{Conflict of Interests}

The authors declare that there is no conflict of interests regarding the publication of this paper.

\section{Acknowledgments}

The authors would like to thank the editor and the anonymous referees for their valuable comments and suggestions that have led to improve the quality of this work.

\section{References}

[1] G. Da Prato and M. Iannelli, "Linear abstract integrodifferential equations of hyperbolic type in Hilbert spaces," Rendiconti del Seminario Matematico dell'Università di Padova, vol. 62, pp. 191-206, 1980.

[2] G. Da Prato and M. Iannelli, "Existence and regularity for a class of integro-differential equations of parabolic type," Journal of Mathematical Analysis and Applications, vol. 112, no. 1, pp. 3655, 1985.

[3] R. C. Grimmer and A. J. Pritchard, "Analytic resolvent operators for integral equations in Banach space," Journal of Differential Equations, vol. 50, no. 2, pp. 234-259, 1983.

[4] A. Lunardi, "Laplace transform methods in integro-differential equations," Journal of Integral Equations, vol. 10, pp. 185-211, 1985.

[5] J. Prüss, "On linear Volterra equations of parabolic type in Banach spaces," Transactions of the American Mathematical Society, vol. 301, no. 2, pp. 691-721, 1987.

[6] J. Prüss, Evolutionary Integral Equations and Applications, Birkhäuser, Basel, Switzerland, 1993.

[7] Ph. Clément and G. Da Prato, "Existence and regularity results for an integral equation with infinite delay in a Banach space," Integral Equations and Operator Theory, vol. 11, no. 4, pp. 480$500,1988$.

[8] R. K. Miller, "Volterra integral equations in a Banach space," Funkcialaj Ekvacioj, vol. 18, no. 2, pp. 163-193, 1975.

[9] G. Chen and R. Grimmer, "Integral equations as evolution equations," Journal of Differential Equations, vol. 45, no. 1, pp. 53-74, 1982.

[10] G. Chen and R. Grimmer, "Semigroups and integral equations," Journal of Integral Equations, vol. 2, no. 2, pp. 133-154, 1980.

[11] W. Desch and R. C. Grimmer, "Initial-boundary value problems for integro-differential equations," Journal of Integral Equations, vol. 10, pp. 73-97, 1985.

[12] W. Desch and W. Schappacher, "A semigroup approach to integro-differential equations in Banach spaces," Journal of Integral Equations, vol. 10, pp. 99-110, 1985.

[13] G. Di Blasio, K. Kunisch, and E. Sinestrari, "Stability for abstract linear functional differential equations," Israel Journal of Mathematics, vol. 50, no. 3, pp. 231-263, 1985.

[14] R. Nagel and E. Sinestrari, "Inhomogeneous Volterra integrodifferential equations for Hille-Yosida operators," in Functional Analysis, vol. 150 of Lecture Notes in Pure and Applied Mathematics, pp. 51-70, Marcel Dekker, New York, NY, USA, 1993.

[15] K. J. Engel and R. Nagel, One-Parameter Semigroups for Linear Evolution Equations, vol. 194 of Graduate Texts in Mathematics, Springer, New York, NY, USA, 2000.
[16] T. Bárta, "Smooth solutions of Volterra equations via semigroups," Bulletin of the Australian Mathematical Society, vol. 78, no. 2, pp. 249-260, 2008.

[17] E. Hille and R. S. Philllips, Functional Analysis and Semigroups, American Mathematical Society Translations Series, 1957.

[18] P. F. Yao, "On the inversion of the Laplace transform of $C_{0}$ semigroups and its applications," SIAM Journal on Mathematical Analysis, vol. 26, no. 5, pp. 1331-1341, 1995.

[19] A. Driouich and O. El-Mennaoui, "On the inverse Laplace transform for $C_{0}$-semigroups in UMD-spaces," Archiv der Mathematik, vol. 72, no. 1, pp. 56-63, 1999.

[20] W. Arendt, C. J. K. Batty, M. Hieber, and F. Neubrander, VectorValued, Laplace Transforms and Cauchy Problems, Birkhäauser, Ulm, Germany, 2010.

[21] I. Cioranescu and V. Keyantuo, "On operator cosine functions in UMD spaces," Semigroup Forum, vol. 63, no. 3, pp. 429-440, 2001.

[22] I. Cioranescu and C. Lizama, "On the inversion of the Laplace transform for resolvent families in UMD spaces," Archiv der Mathematik, vol. 81, no. 2, pp. 182-192, 2003.

[23] M. Haase, "The complex inversion formula revisited," Journal of the Australian Mathematical Society, vol. 84, no. 1, pp. 73-83, 2008.

[24] C. Lizama and J. C. de Souza, "The complex inversion formula in UMD spaces for families of bounded operators," Applicable Analysis, vol. 91, no. 5, pp. 937-946, 2012.

[25] L. F. Ho and D. L. Russell, "Admissible input elements for systems in Hilbert space and a Carleson measure criterion," SIAM Journal on Control and Optimization, vol. 21, no. 4, pp. 614-640, 1983.

[26] G. Weiss, "Admissibility of unbounded control operators," SIAM Journal on Control and Optimization, vol. 27, no. 3, pp. 527-545, 1989.

[27] G. Weiss, "Two conjectures on the admissibility of control operators," in Estimation and Control of Distributed Parameter Systems, F. Kappel and W. Desch, Eds., pp. 367-378, Birkhäuser, Basel, Switzerland, 1989.

[28] B. Jacob and J. R. Partington, "The Weiss conjecture on admissibility of observation operators for contraction semigroups," Integral Equations and Operator Theory, vol. 40, no. 2, pp. 231243, 2001.

[29] C. Le Merdy, “The Weiss conjecture for bounded analytic semigroups," Journal of the London Mathematical Society, vol. 67, no. 3, pp. 715-738, 2003.

[30] B. Jacob and J. R. Partington, "Admissibility of control and observation operators for semigroups: a survey," in Current Trends in Operator Theory and Its Applications, vol. 149, pp. 199221, Springer, New York, NY, USA, 2004.

[31] O. Staffans, Well-Posed Linear Systems, vol. 103 of Encyclopedia of Mathematics and Its Applications, Cambridge University Press, Cambridge, UK, 2005.

[32] F. Maragh, H. Bounit, A. Fadili, and H. Hammouri, "On the admissible control operators for linear and bilinear systems and the Favard spaces," Bulletin of the Belgian Mathematical Society-Simon Stevin, vol. 21, no. 4, 2014.

[33] M. Jung, "Admissibility of control operators for solution families to Volterra integral equations," SIAM Journal on Control and Optimization, vol. 38, no. 5, pp. 1323-1333, 2000.

[34] B. Jacob and J. R. Partington, "Admissible control and observation operators for Volterra integral equations," Journal of Evolution Equations, vol. 4, no. 3, pp. 333-343, 2004. 
[35] B. Jacob and J. R. Partington, "A resolvent test for admissibility of Volterra observation operators," Journal of Mathematical Analysis and Applications, vol. 332, no. 1, pp. 346-355, 2007.

[36] B. H. Haak, B. Jacob, J. R. Partington, and S. Pott, "Admissibility and controllability of diagonal Volterra equations with scalar inputs," Journal of Differential Equations, vol. 246, no. 11, pp. 4423-4440, 2009.

[37] A. Fadili and H. Bounit, "On the Favard spaces and the admissibility for Volterra systems with scalar kernel," Under review.

[38] H. Bounit, A. Driouich, and O. El-Mennaoui, "Admissibility of control operators in UMD spaces and the inverse Laplace transform," Integral Equations and Operator Theory, vol. 68, no. 4, pp. 451-472, 2010.

[39] E. G. Bajlekova, Fractional Evolution Equations in Banach Spaces, Technische Universiteit Eindhoven, Eindhoven, The Netherlands, 2001.

[40] W. Arendt and J. Prüss, "Vector-valued Tauberian theorems and asymptotic behavior of linear Volterra equations," SIAM Journal on Mathematical Analysis, vol. 23, no. 2, pp. 412-448, 1992.

[41] W. Desch and J. Prüss, "Counterexamples for abstract linear Volterra equations," Journal of Integral Equations and Applications, vol. 5, no. 1, pp. 29-45, 1993.

[42] J. Bourgain, "Some remarks on Banach spaces in which martingale difference sequences are unconditional," Arkiv för Matematik, vol. 21, no. 2, pp. 163-168, 1983.

[43] J. Bourgain, "Vector-valued singular integrals and the $H^{1}$-BMO duality," in Probability Theory and Harmonic Analysis, vol. 98 of Textbooks Pure and Applied Mathematics, pp. 1-19, 1986.

[44] D. L. Burkholder, "Martingales and singular integrals in Banach spaces," in Handbook of the Geometry of Banach Spaces, pp. 233269, North-Holland, Amsterdam, The Netherlands, 2001.

[45] M. Haase, New Tends in the Theory of Hyperbolic Equations, Birkhäauser, Boston, Mass, USA, 2000.

[46] H. Amann, Linear and Quasilinear Parabolic Problems, Birkhäauser, Boston, Mass, USA, 1995.

[47] A. Pazy, Semigroups of Linear Operators and Applications to Partial Differential Equations, vol. 44 of Applied Mathematical Sciences, Springer, New York, NY, USA, 1983.

[48] R. Grimmer and J. Prüss, "On linear Volterra equations in Banach spaces," Computers \& Mathematics with Applications, vol. 11, no. 1-3, pp. 189-205, 1985. 


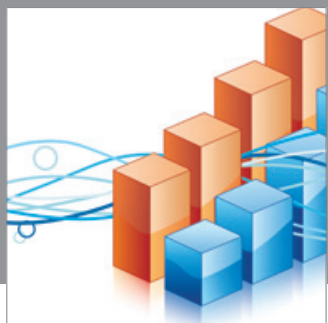

Advances in

Operations Research

mansans

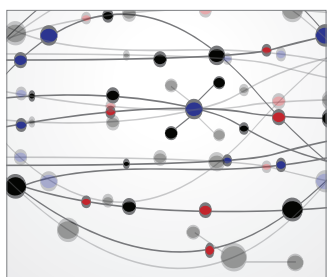

The Scientific World Journal
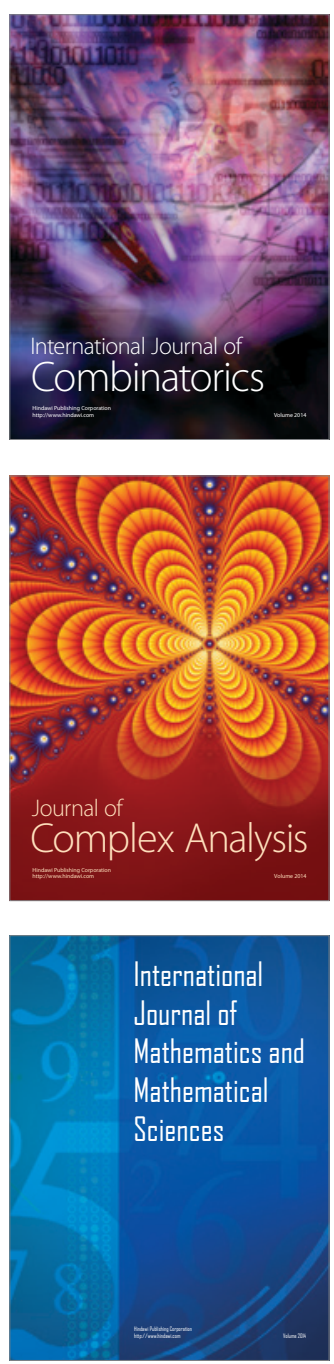
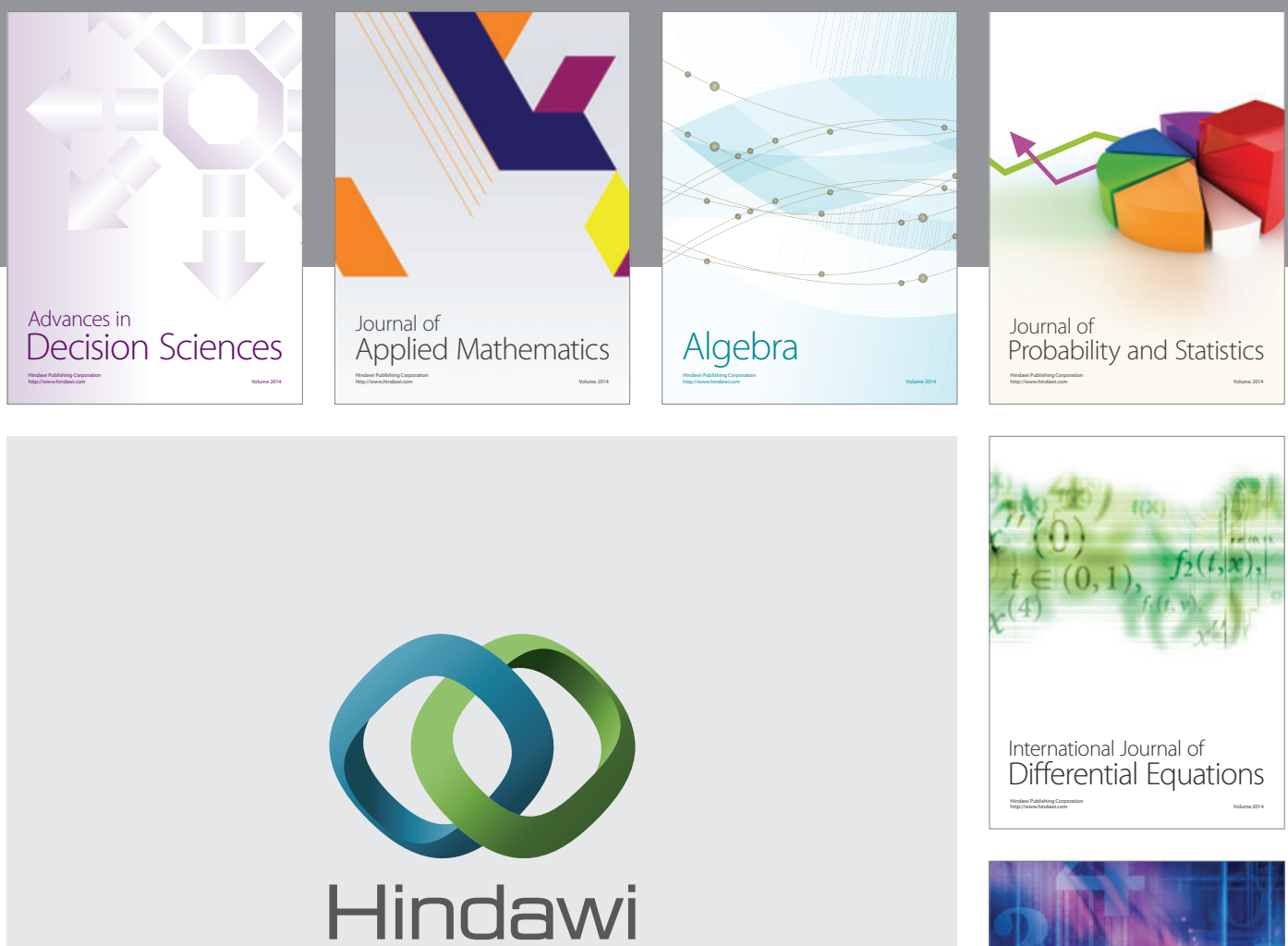

Submit your manuscripts at http://www.hindawi.com
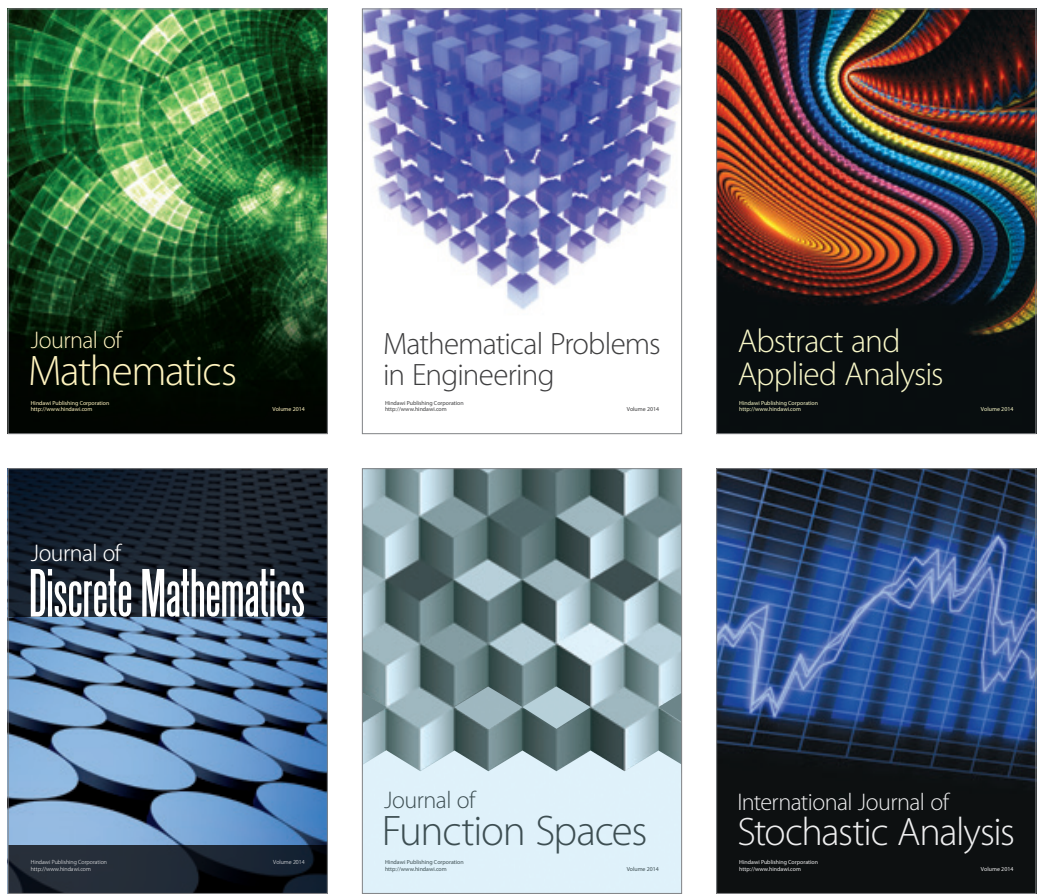

Journal of

Function Spaces

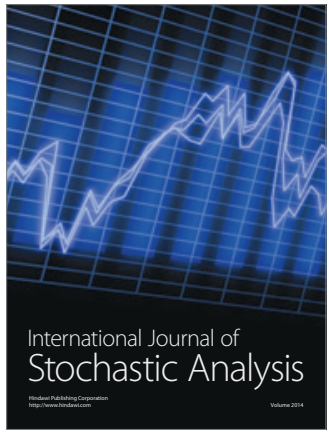

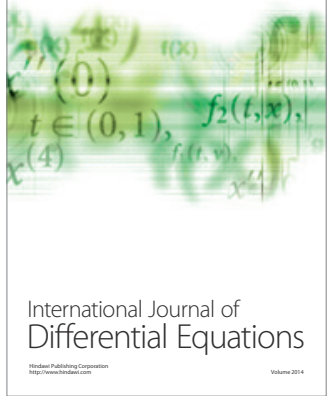
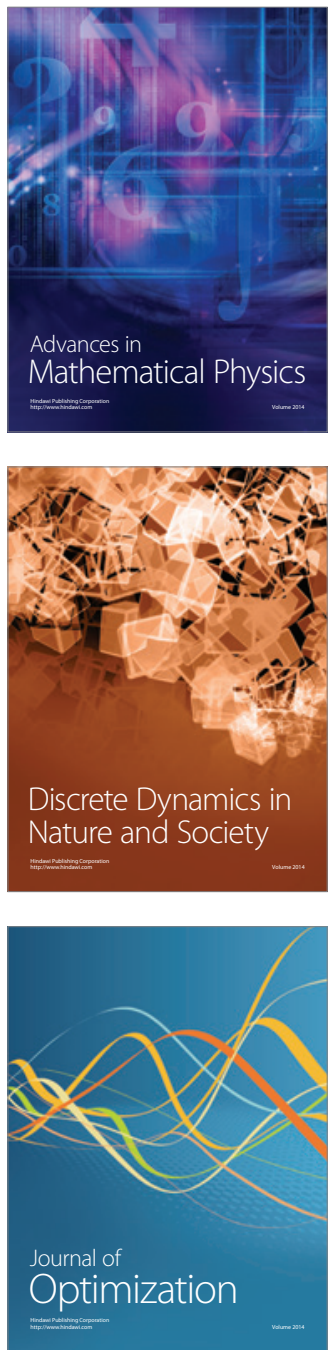